\title{
Presynaptic Depression in Phasic Motor Nerve Terminals and Influence of 5-HT on Vesicle Dynamics
}

\author{
A.F.M. Johnstone, S.S. Kellie and R.L. Cooper* \\ Department of Biology, University of Kentucky, Lexington, KY 40506-0225, USA
}

\begin{abstract}
Synaptic depression that is induced by electrical stimulation of the glutamatergic neuromuscular junction (NMJ) of the crayfish can be offset by recruitment of vesicles from a presynaptic reserve pool. This recruitment occurs following treatment of the NMJ with serotonin (5-HT), which results in a delay in the onset of synaptic depression induced by high frequency stimulation. The results of this study demonstrate that the releasable vesicles are insufficiently replenished during high frequency stimulation and that the readily releasable pool of vesicles (RRP) can be enhanced by the reserve pool (RP) in the presence of 5-HT. Anatomical visualization of vesicular pools by transmission electron microscopy after depression or during 5-HT treatment showed no differences in the number of docked and RRP vesicles. We propose that the RRP vesicles can recycle empty and that a role for 5-HT might be to induce a rapid enhancement of synaptic transmission during synaptic fatigue.
\end{abstract}

\section{INTRODUCTION}

Synaptic depression is a recognized physiological phenomenon within the nervous system. However, the detailed cellular mechanisms and modulation of the processes are not yet fully understood. Synaptic depression is often defined as a use-dependent long-lasting decrease in synaptic strength [1]. Evidence in some models point to a presynaptic component, whereas in others a postsynaptic role may dominate. Depending on the model preparation used it can be challenging to determine the mechanistic determinates of synaptic depression because of the experimental limitations of measuring directly at synapses. Because synaptic depression plays a role in memory and behavior, it is important to investigate the mechanisms underlying synaptic depression. Our hope is that knowing how to modulate the synaptic responses in defined simple systems will allow an overall basic understanding to prevail for most other synaptic models. The neuromuscular junction (NMJ) in the crayfish offers many advantages because one to a few excitatory motor neurons innervate a muscle, and the neurons are identifiable [2]. The excitatory neurotransmitter at these NMJs is glutamate and the excitatory postsynaptic potentials (EPSP) are graded. Thus, the biophysical properties of graded events are analogous to those on dendrites of neurons within the CNS of vertebrates [3].

Modulation of the rate of synaptic depression and the recovery process opens the door to deciphering the mechanisms of synaptic depression. For these purposes, we made use of the crayfish leg extensor preparation and the exogenous application of 5-hydroxytryptamine (5-HT). This leg extensor preparation provides several experimental advantages because individual muscle fibers are innervated by both phasic and tonic motor neurons. Repetitive $5 \mathrm{~Hz}$ stimulation of the phasic nerve gives rise to large excitatory post

*Address correspondence to this author at the Department of Biology, University of Kentucky, Lexington, KY 40506-0225, USA;

E-mail: RLCOOP1@email.uky.edu synaptic potentials (EPSPs) that become greatly depressed after several minutes [4]. At the crayfish NMJ, 5-HT has been shown to induce an inositol triphosphate (IP3) cascade [5], and evidence suggests that that bathing the preparation in a 5-HT-containing solution causes a facilitated release of neurotransmitter-containing vesicles from the RRP $[6,7,8]$. Because 5-HT increases the probability of release by evoked stimulation [9], we postulated that the rate of synaptic depression would be offset during evoked stimulation in the presence of 5-HT.

To determine whether synaptic depression is due to a limit in the number of releasable vesicles, the depressed nerve terminals that were not exposed to 5-HT during the stimulation period were later exposed when they were drastically depressed under continuous electrical stimulation. This addressed if 5-HT could recruit vesicles from the reserve pool in depressed terminals. Given that the majority of a 5HT-induced response is mediated by IP3 the availability of ATP is also indirectly examined within this experimental paradigm as being a potential limiting factor for vesicle fusion within depressed nerve terminals. Since ATP is required for vesicular fusion and if ATP is a limiting factor, 5-HT should not have an effect on depressed terminals.

\section{MATERIALS AND METHODOLOGY}

\section{Animals}

Mid-sized crayfish (Procambarus clarkii), measuring 6$10 \mathrm{~cm}$ in body length, were obtained from Atchafalaya Biological Supply Co. (Raceland, Louisiana. The crayfish were housed in individual tanks that were located in an aquatic facility within the laboratory. They were fed fish food pellets every three days. Only male crayfish in their intermolt stage were used.

\section{Muscle-Nerve Preparation}

The extensor muscle of the first walking legs was prepared using the method described by Bradacs [10]. The preparation was pinned out in a Slygard dish for viewing 
with a Nikon, Optiphot-2 upright fluorescent microscope using a $40 \mathrm{X}$ (0.55 NA) Nikon water immersion objective. The dissected preparations were maintained in crayfish saline, a modified Van Harreveld's solution $(205 \mathrm{mM} \mathrm{NaCl}$; 5.3mMKCl; $13.5 \mathrm{mM} \mathrm{CaCl}_{2} 2 \mathrm{H}_{2} \mathrm{O} ; 2.45 \mathrm{mM} \mathrm{MgCl} 26 \mathrm{H}_{2} \mathrm{O}$; $0.5 \mathrm{mM}$ HEPES adjusted to $\mathrm{pH} 7.4$ ) at $20^{\circ} \mathrm{C}$.

\section{Evoked Post-Synaptic Potentials (EPSPs)}

Intracellular muscle recordings were obtained with a $3 \mathrm{M}$ $\mathrm{KCl}$-containing microelectrode placed in a muscle fiber. A Grass S-88 stimulator and stimulus isolation unit with leads to a standard suction electrode set-up were used. EPSPs were measured following stimulation of the preparation at $0.5 \mathrm{~Hz}$ and $5 \mathrm{~Hz}$ frequencies. Selective stimulation of the excitatory axon was carried out using a 'macro-patch' electrode with an inner diameter of 15 to $20 \mu \mathrm{m}$ placed directly on the phasic axon [10]. The excitatory axon is identified easily after staining with the vital mitochondrial stain 4-Di-2-ASP, because of the large number of mitochondria contained therein [11].

When the EPSP amplitude is reduced to just being undetectable over the baseline $(<0.5 \mathrm{mV})$ we deemed this as a depressed synaptic response. After induction of synaptic depression, ten minutes was allowed to elapse before repeating the $0.5 \mathrm{~Hz}$ stimulation for five events followed by a continuous $5 \mathrm{~Hz}$ stimulation. In some of the preparations, 5-HT (100 $\mathrm{nM}$ ) was added to the bath during the ten minute rest period after inducing synaptic depression. In other preparations, 5-HT (100 nM) was applied before the initial depression was induced to examine if the rate of depression was altered. To assess whether the properties of the muscle had changed after synaptic depression occurred in the phasic motor neuron, the EPSPs of the tonic motor neuron were monitored within the same muscle fiber. The percentage difference in EPSP responses was calculated as the absolute difference (initial - experimental) divided by "initial" and multiplied by 100 . For example, a $2 \mathrm{mV}$ response changing to a $10 \mathrm{mV}$ response produces a $400 \%$ change.

Responses were recorded with a standard intracellular electrode amplifier (AxoClamp 2A, Axon Instruments). Electrical signals were recorded on-line to a Power Mac 9500 via a MacLab/4s interface. EPSPs were acquired at either $10 \mathrm{kHz}$ or $20 \mathrm{kHz}$.

\section{Application of Neuromodulators}

To apply exogenous compounds to the preparation, the bathing medium was rapidly exchanged with saline containing 5-HT (Sigma). Solutions were made fresh before each experiment from a frozen standard (10 mM 5-HT in saline).

\section{Transmission Electron Microscopy (TEM)}

All preparations were fixed in a $2.5 \%$ gluteraldehyde, $0.5 \%$ formaldehyde buffered solution $(0.1 \mathrm{M}$ sodium cacodylate, $0.022 \%$ wt. $\mathrm{CaCl}_{2}, 4 \%$ wt. sucrose, and adjusted to $\mathrm{pH}$ 7.4) and changed twice during one hour with two changes. The tissue was post fixed with a $2 \%$ osmium tetroxide buffered solution and finally embedded in Eponate 812. The samples were then thin-sectioned serially on a Reichert ultracut E microtome, picked up with slotted grids and these grids were laid on formvar for drying. The grids were then stained with uranyl acetate and lead citrate. The stained sections were viewed on a Phillips FEI Tecnai, Bio Twin 12 model transmission electron microscope at $80 \mathrm{kV}$.
In preparations to examine vesicle location, while stimulation proceeded, the fixation was immediately performed in the recording dish with only delay from the time to remove the ground wire to adding the fix ( $\sim 1$ second). The fixative solution was added directly over the preparation and the bathing media was replaced 3 times to insure good penetration of the tissue. Later each recording dish, stimulating and recording electrodes were discarded, but this was necessary to insure the most rapid fixation process within the dish after induction of synaptic depression without spending time to remove electrodes.

\section{Measurements for Vesicle Location and Distribution}

Each transmission electron micrograph that contained a synapse was cataloged. In some cases, serial sections of a complete synapse were obtained. The presynaptic terminals were classified as excitatory by the shape of the vesicles contained therein. Inhibitory terminals contain obliquely shaped vesicles [12] and were not further used for quantification. The presence of dense bodies associated with synapses within the presynaptic terminal was used to define an active zone (AZ). In the AZ, vesicles normally cluster with the presynaptic membrane [13]. These dense bodies are thought to serve as attachment points in the cytoskeleton to deliver tethered synaptic vesicles to the AZ possibly from reserve and recycling pools.

Synapses at the crayfish NMJ do not have a grid of AZs. Instead, they show synaptic variation such that some synapses may contain only a single AZ, whereas others might have multiple AZs whose distances from one another vary $[13,14]$. We sought to accurately measure vesicles that are docked at the synapse and RRP by serially sectioning the synapses around single AZs, thereby insuring that the counts were not skewed by vesicles associated with neighboring AZs. Thus, three sections on either side of an AZ of interest were viewed, and only sections within one section to either side of the section that contained an AZ were used for anatomical measurements of vesicle pools. In the crayfish opener NMJ preparation, the dense bodies of the excitatory terminals are viewed as hemispheres of about $60 \mathrm{~nm}$ in diameter and sit within the cross-section of a hemisphere that faces the synapse [15]. This is documented by the occasional face on view in parallel sectioning of synapses [16]. Thus, in cross-sections of $75 \mathrm{~nm}$ thickness a dense body can be contained within a single section. If the dense body is seen within two sections, then sections on either side of these sections were also used for analysis (see results section). Care was taken to avoid stereological errors in measuring objects using TEM from 2-D images of 3-D tissue (see Results).

\section{Statistical Analysis}

Numerical data were presented as mean \pm standard error of the mean (SEM). When the basic assumptions of the parametric Student's $t$-test were valid, this test was used. Otherwise, the non-parametric Wilcoxon rank sum test was used.

\section{RESULTS}

The phenotype of the extensor muscle fibers in the crayfish leg has previously been mapped for regional differences from proximal to distal and lateral to medial [10]. It has also been established previously that all the muscle fibers in the 
extensor muscle are innervated by both the phasic and tonic excitatory nerves as well as an inhibitory nerve. Because the centrally located muscle fibers show pronounced tonic and phasic EPSPs, centrally located muscles were used in this study to investigate the depression of the phasic terminals and the role of 5-HT in offsetting synaptic depression (Fig. 1). The EPSPs that were produced by tonic nerve stimulation were not detectable with a single stimulus. However, when a train of pulses were given to induce short-term facilitation, the EPSPs became enhanced (Fig. 1).

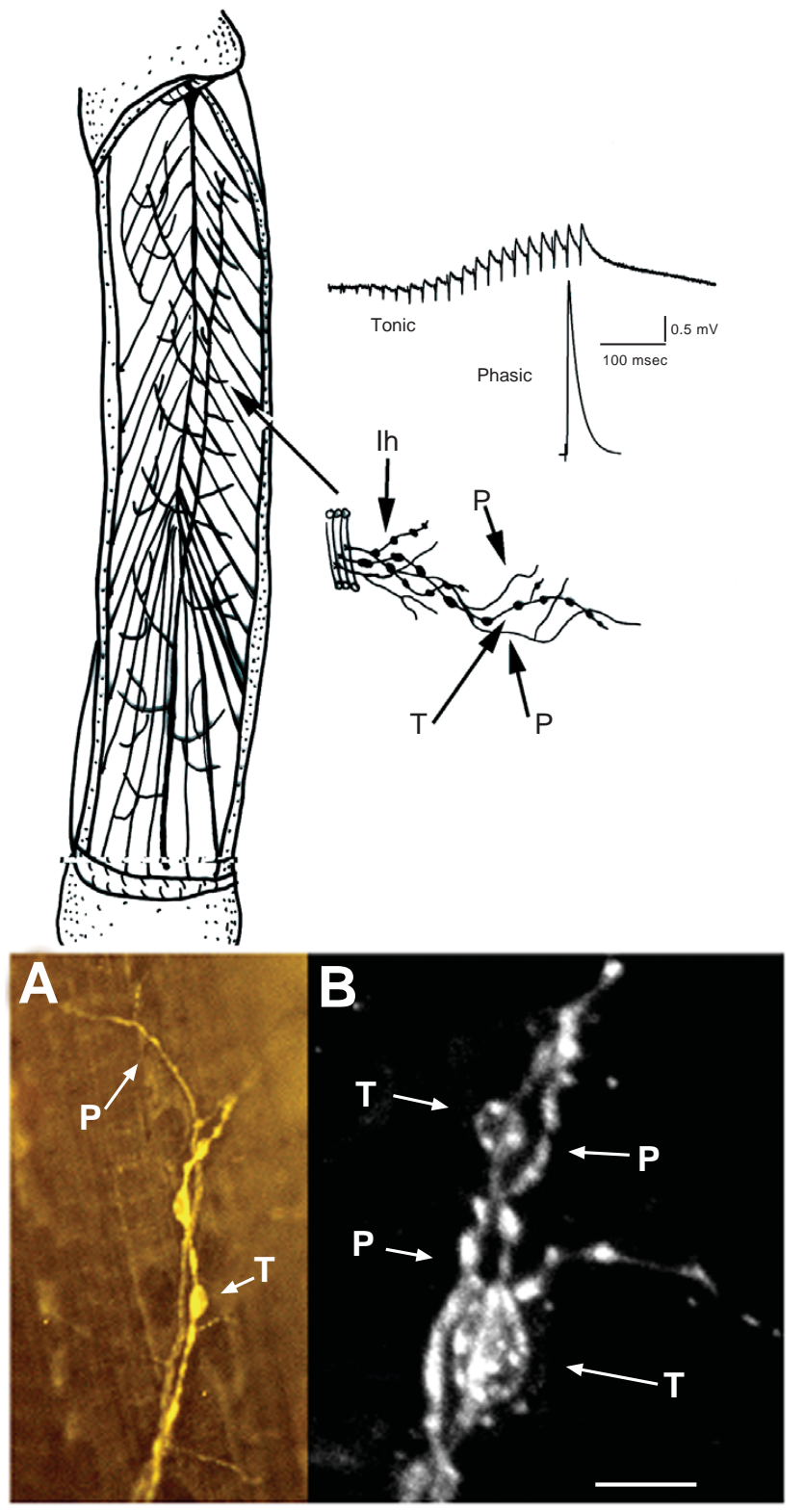

Fig. (1). Anatomy of extensor muscle neuron innervation: Top: Diagram depicting the extensor muscle preparation and tonic ( $\mathrm{T}$, largest), phasic (P) and inhibitor (Ih, smallest) innervation (insert). Each muscle fiber of the extensor is innervated by each (T, P and Ih). Top: The low output NMJ must be facilitated to detect the EPSPs (Tonic), whereas the high output produces a large response with a single stimulus (Phasic). Bottom: A: Phasic and tonic extensor innervation stained with 4-Di-2-Asp. B: Anti-synaptotagmin antibody staining of a depressed phasic NMJ showing punctuate staining of vesicle pools in both tonic and phasic NMJs (scale bar $10 \mu \mathrm{m})$. The top panel is also shown in Cooper [6].
The Properties of Synaptic Depression and the Influence of 5-HT

When the phasic axon was stimulated at a low frequency $(0.5 \mathrm{~Hz})$, the EPSPs did not facilitate. However, there was a pronounced facilitation that led to a depressed response over time at a higher stimulation frequency $(5 \mathrm{~Hz})$. A representative profile for the EPSP amplitudes during the stimulation paradigm of an initial $0.5 \mathrm{~Hz}$ followed by a $5 \mathrm{~Hz}$ for a prolonged duration to induce depression is shown in Fig. (2). After the responses were fully depressed, the stimulation was stopped. At this point, the bathing medium was replaced either three times with normal saline or with a solution containing 5-HT (100 $\mathrm{nM})$ and the preparation was left to recover for ten minutes before repeating the stimulation paradigm of $0.5 \mathrm{~Hz}$ and $5 \mathrm{~Hz}$. Only five EPSPs at $0.5 \mathrm{~Hz}$ were recorded before increasing to $5 \mathrm{~Hz}$. To index differences among the treatment groups (i.e., saline only; exposure to 5HT throughout stimulation; 5-HT exposure after the initial EPSP depression), four time parameters were measured (shown in Fig. 2), namely, (i) the time from the initiation of the $5 \mathrm{~Hz}$ stimulation to the peak of the facilitated response; (ii) the time to the onset of decay; (iii) the time from the onset of the decay to the time at which the EPSP amplitude had decayed to $50 \%$ of the maximum response in the first stimulation phase; (iv) a second decay response from the peak of the recovery response to $50 \%$ in amplitude during the second stimulation paradigm.

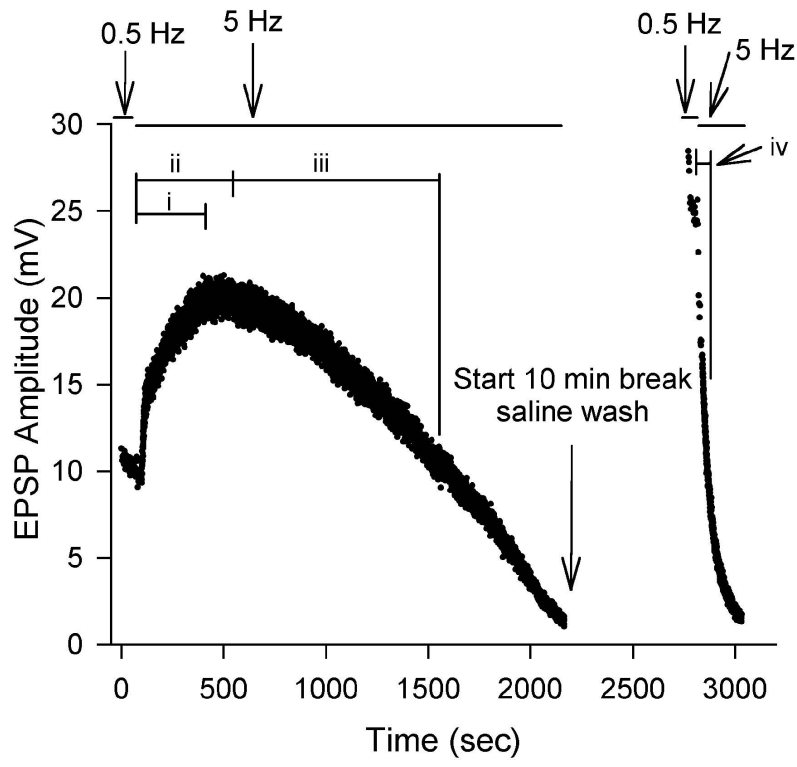

Fig. (2). Depression stimulation paradigm: EPSP amplitude (mV) under separate stimulation parameters. i-iii: Stimulation increase from $0.5 \mathrm{~Hz}$ to $5 \mathrm{~Hz}$, which quickly starts to depress the phasic nerve. iv: After the phasic terminal is depressed, a 10 minute saline wash is carried out; once the stimulation returns, depression occurs more quickly due to the previous stimulation.

Assuming that the depression was primarily a presynaptic phenomenon, we set out to determine whether 5-HT would result in enhanced recovery after synaptic depression occurred by bathing the preparation in 5 -HT $(100 \mathrm{nM})$ during the 10 minute resting period (Fig. 3). Because the results of previous studies $[17,18,19]$ indicated that 5 -HT promotes vesicle docking and increases the probability of release, we 
predicted that a more pronounced recovery would occur. In addition, one would assume that depression would be offset during the high frequency stimulation when the preparation was bathed in 5-HT during the entire stimulation paradigm. Hence, the results for the influence of 5-HT were compared with the results obtained in preparations that were exposed only to saline with the same stimulation paradigms $(n=5$ in all three conditions).
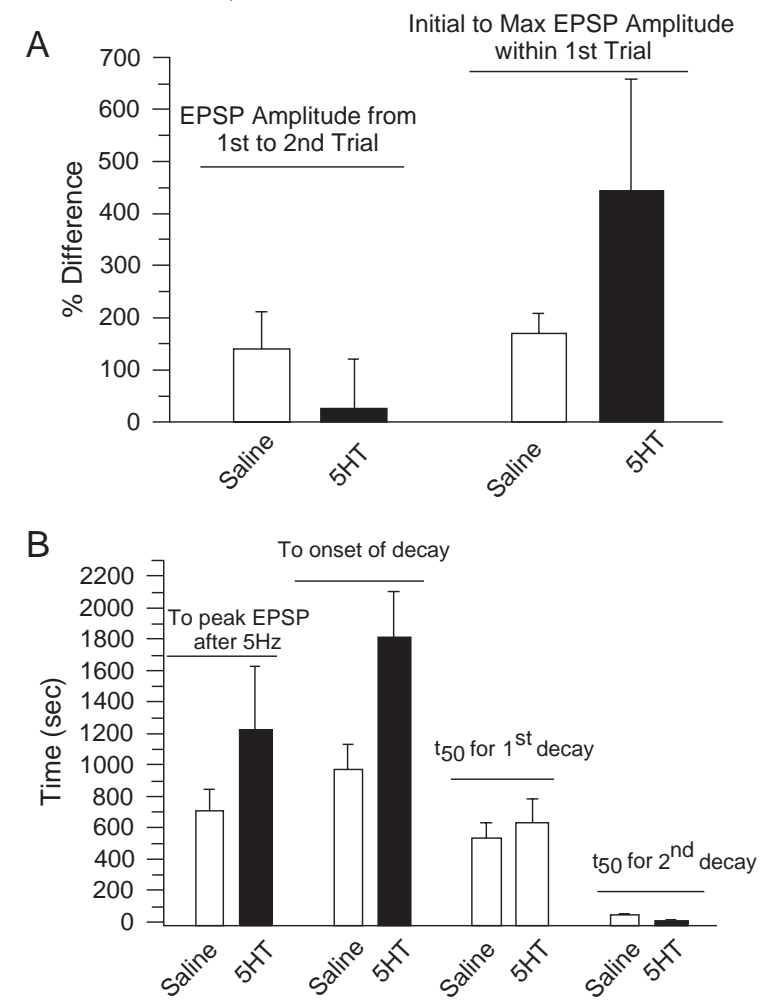

Fig. (3). EPSP Percent and time differences: A: EPSP amplitude percent difference from 1 st to 2 nd trial. The addition of 5-HT greatly reduces the EPSP percentage between trials, but increases the max EPSP with in the 1st trial, suggesting two vesicle pools are active, but under separate control mechanisms. B: Time to peak EPSP, decay and time to $50 \%$ decay for the 1st and 2nd trials in saline and in presence of 5-HT. 5-HT increases the time to peak EPSP amplitude and the onset of decay, but decreases the time for $50 \%$ decay for the 1 st and 2nd trials once depression starts, suggesting that 5-HT causes an initial increase in transmitter release, but a slower recovery rate after the onset of depression (Fig. 2).

The amplitudes of the EPSPs were monitored at various time points throughout the entire paradigm for the three experimental conditions. Because the initial EPSP amplitudes varied in each preparation, a percent change in the amplitude was used to make the comparisons. The percent difference in the amplitudes of the EPSPs during the initial $0.5 \mathrm{~Hz}$ stimulation and the $0.5 \mathrm{~Hz}$ stimulation after a ten minute rest was determined. Likewise, the percent difference in EPSP amplitude from the $0.5 \mathrm{~Hz}$ initial stimulation to the maximum facilitated response was determined within the first stimulation trial. The percent differences suggested that 5-HT produced a larger peak response when compared with that obtained in saline-exposed preparations during the first stimulation phase $(\mathrm{p}<0.05)$. Exposure to 5-HT after the initial depression did not produce a marked recovery from the depressed state.
However, exposure to 5-HT throughout the entire stimulation conditions produced a reduction in recovery from depression when compared with those obtained after saline exposure (Fig. 3A). This is likely due to the use of RRP and RP vesicles. Exposure to 5-HT did produce a larger peak response during the initial facilitation of $5 \mathrm{~Hz}$ stimulation when compared with that observed in saline-exposed preparations ( $\mathrm{p}<0.05$, Fig. 3A-far right column).

The differences in the indices are shown in Fig. 3B (the various time points are shown in Fig. 2). These are: (1) the time to reach a maximum response; (2) the onset of decay; (3) the decay rate. When the preparations were bathed in 5-HT before starting the routine stimulation paradigm, the time to a peak response was prolonged $(p<0.05$, Fig. 3B) when compared with that determined for saline only exposed preparations. During the second stimulation paradigm, the preparations exposed to 5-HT throughout the entire period depressed faster than those only exposed to saline or 5-HT after the initial depression.

\section{Nerve Terminal Profiles}

The phasic terminals are easily identified by their filiform morphology when compared with the tonic terminals that contain swellings (i.e., varicosities). The synaptic locations, where vesicles cluster, were observed by immunocytochemical staining with the antibody to synaptotagmin (Fig. 1B) [20]. Patches were present within the large varicosities
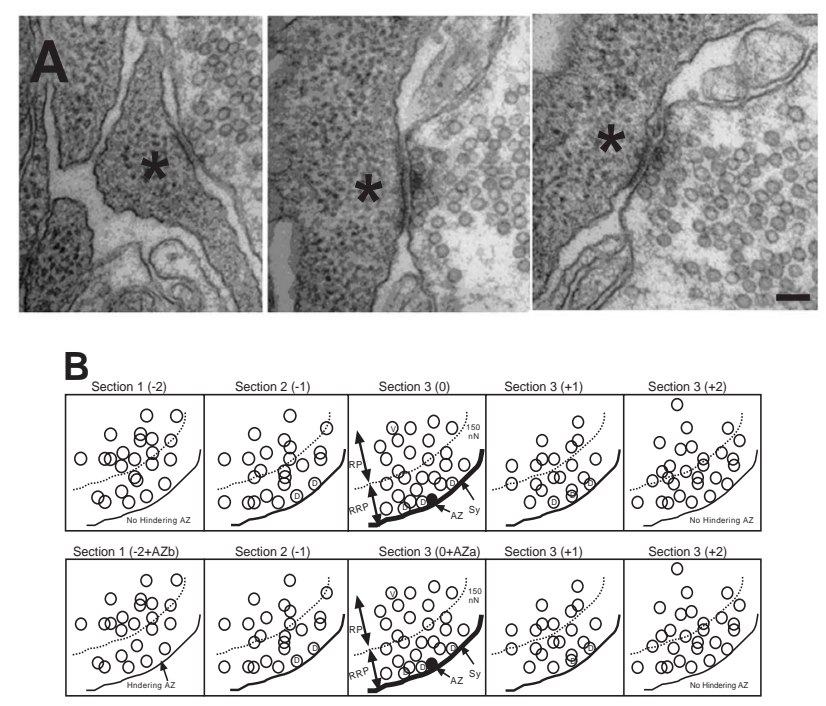

Fig. (4). TEM Analysis of RRP and docked vesicles: The readily releasable pool and docked vesicles were quantified visually by serial TEM (* Denotes same synapse for each section and these would align in a serial stack of sections). A. An example TEM serial sections that would be used for vesicle quantification, in this case, a control (no 5-HT). B: (upper panel) Diagram depicting a serial sections that would be used. An AZ (demarked as section "0"), a section in front (demarked "-1") and behind (demarked " $+1 ")$ were used in the quantification. Sections -2 and +2 were also inspected to insure there were no neighboring AZ's. Vesicles that were in direct contact with the synapse were counted as docked, while any vesicles within $150 \mathrm{~nm}$ of the synapse were counted as the RRP. Any vesicles beyond that was considered to be the RP and not counted. B: (lower panel) Diagram depicting serial sections that would not be used due to neighboring active zones (AZ). 
that were related to the synaptic structure observed in these terminals $[10,21,22]$. It was also obvious that there were patches, along the length of the phasic terminals, which correlated with the synaptic domains [22]. Some preparations were immediately fixed after induction of synaptic depression and stained with the antibody to synaptotagmin. There were no detectable differences between the punctate patterns of depressed terminals and non-depressed phasic terminals.

The phasic nerve terminals were visualized by TEM so that the RRP and docked vesicles could be quantified (Fig. 4A and 4B). Using the method described above, the docked and RRP vesicles were quantified from three samples of each experimental paradigm. The control group (denoted as "Control" in Table 1) was stimulated at $5 \mathrm{~Hz}$ until it depressed. The first group was stimulated at $5 \mathrm{~Hz}$ until depression occurred and then 5 -HT was added $(1 \mu \mathrm{M})$ (denoted as $\mathrm{D} \rightarrow 5-\mathrm{HT}$ on Table 1). The second experimental group was stimulated at $5 \mathrm{~Hz}$ in the presence of 5-HT until depression occurred (denoted as 5-HT in Table 1). No difference was observed in the distribution of RRP and docked vesicles due to the $\mathrm{D} \rightarrow 5-\mathrm{HT}$ paradigm. A $31 \%$ decrease in the RRP and a $60 \%$ decrease in docked vesicles were observed in the 5-HT treatment group (Table 1).

\section{DISCUSSION}

This study demonstrated that the RP and RRP of vesicles within a phasic motor nerve terminal in the crayfish extensor muscle preparation are identifiable by physiological and neuromodulatory means. This was approached by evaluating the active status of the vesicles within the nerve terminal. Here, we depressed the phasic nerve terminal with high stimulation frequency $(5 \mathrm{~Hz})$ in the absence or presence of 5-HT while monitoring EPSPs with intracellular muscle recordings. Given the results, we suggest that there are two operative (functional) pools of vesicles. One pool is active during high stimulation frequency and can be depleted rapidly. The second pool can be recruited by 5-HT which acts to prolong the onset of depression by increasing the time to reach maximal EPSP amplitude.

Table (1). RRP and Docked vesicle analysis: Vesicle counting data: Using the method described, docked and readily releasable pool (RRP) vesicles were quantified from three samples of each parameter. 1. Controls: Stimulation at $5 \mathrm{~Hz}$ until depression was observed. 2. Depression to 5-HT $(\mathrm{D} \rightarrow 5 \mathrm{HT})$ : Stimulation at $5 \mathrm{~Hz}$ until depression was observed, after which 5 -HT $(1 \mu \mathrm{M})$ was added to the preparation. 3. 5-HT on preparation whole time (5-HT): Preparations were in the presence of 5 -HT $(1 \mu \mathrm{M})$ during the whole stimulation until depression was observed (onset of depressed delayed but once started depression was quick). Preliminary data between the controls and D $\rightarrow 5-\mathrm{HT}$ showed little to no difference as compared to the 5-HT exposure group in which there was a $31 \%$ decrease in the RRP and a $60 \%$ decrease in docked vesicles.

This second pool can be quickly depleted once synaptic depression starts. When a terminal is depressed the state of depression is maintained for a prolonged time. Because the muscle fiber could still be stimulated by a tonic motor neuron while the phasic terminal is depressed, this demonstrates that the muscle is still responsive and that synaptic depres-
Table 1. RRP and Docked Vesicle Analysis

\begin{tabular}{llll} 
Preparation & Order & RRP & Docked \\
\hline Control 1 & -1 & 13 & 5 \\
& 0 & 15 & 5 \\
& +1 & $\underline{11}$ & 4 \\
\hline
\end{tabular}

\begin{tabular}{llll} 
Mean & & $\mathbf{1 3}$ & $\mathbf{5}$ \\
\hline Control 2 & -1 & 13 & 3 \\
& 0 & 17 & 4 \\
& +1 & $\underline{11}$ & 2
\end{tabular}

\begin{tabular}{llll} 
Mean & & $\mathbf{1 4}$ & $\mathbf{3}$ \\
\hline Control 3 & 1 & 23 & 5 \\
& 0 & 21 & 7 \\
& +1 & $\underline{16}$ & 6
\end{tabular}

\begin{tabular}{llll} 
Mean & & $\mathbf{2 0}$ & $\mathbf{6}$ \\
Cumulative Mean: & & $\mathbf{1 6}$ & $\mathbf{5}$ \\
Preparation & Order & RRP & Docked \\
\hline D $\rightarrow$ 5HT 1 & -1 & 14 & 4 \\
& 0 & 12 & 5 \\
& +1 & $\underline{10}$ & 14 \\
& &
\end{tabular}

\begin{tabular}{llll} 
Mean & & $\mathbf{1 2}$ & $\mathbf{8}$ \\
\hline D 5HT 2 & -1 & 7 & 1 \\
& 0 & 23 & 4 \\
& +1 & $\underline{10}$ & 5
\end{tabular}

\begin{tabular}{llll} 
Mean & & $\mathbf{1 3}$ & $\mathbf{3}$ \\
\hline D $\rightarrow$ 5HT 3 & 1 & 24 & 4 \\
& 0 & 23 & 4 \\
& +1 & $\underline{18}$ & 4
\end{tabular}

\begin{tabular}{llll} 
Mean & & $\mathbf{2 2}$ & $\mathbf{4}$ \\
Cumulative Mean: & & $\mathbf{1 6}$ & $\mathbf{5}$ \\
Preparation & Order & RRP & Docked \\
\hline 5HT 1 & -1 & 9 & 2 \\
& 0 & 13 & 2 \\
& +1 & $\underline{17}$ & 2
\end{tabular}

\begin{tabular}{llll} 
Mean & & $\mathbf{1 3}$ & $\mathbf{2}$ \\
\hline 5HT 2 & -1 & 10 & 2 \\
& 0 & 10 & 1 \\
& +1 & $\underline{8}$ & 2
\end{tabular}

\begin{tabular}{cccc} 
Mean & & 9 & 2 \\
\hline \multirow{5}{*}{ HHT 3 } & -1 & 10 & 2 \\
& 0 & 11 & 2 \\
& +1 & $\underline{11}$ & 3
\end{tabular}

\begin{tabular}{lll} 
Mean & 11 & 2 \\
\hline Cumulative Mean: & 11 & 2
\end{tabular} 
sion occurs only in relation to the phasic NMJs. To investigate the potential association of docked and RRP vesicle pools in relation to synaptic depression, the phasic nerve terminals were examined using TEM. However, there were no observable differences in the proportions of RRP and docked vesicles in depressed terminals (Fig. 4 and Table 1). Thus, the RRP can be depleted faster, as measured electrophysiologically, in the presence of 5-HT because of the increased release probability during the exposure. But, in addition, there are reserve pools (RP) of vesicles besides the RRP which are likely recruited into the RRP by 5-HT; however, we did not observe anatomically an enhancement of docked vesicles due to 5-HT exposure.

The criterion of anatomical measures of RRP close to the synapse was shown not to be of great value because the demarcation is relative. However, the criterion does serve as one approach to indexing changes in the population. Using special labeling techniques at the frog NMJ, Rizzoli and Betz [23] noted that vesicles within the RRP have specific identities and that about $30 \%$ of recycled vesicles were lost to the RP. In addition, they demonstrated that vesicles in the RRP could arise from the edges of vesicle clusters which would fall outside a $150 \mathrm{~nm}$ distance from the synapse. In such a case this would not fit the RRP criterion we set for indexing the pools. Utilizing novel approaches to assess docked vesicles held at the AZ possible waiting to be released [24], as compared to rapid recycling "kiss and run" vesicular events [25] could possible help to deifier mechanisms of synaptic fatigue/depression.

The proposal that vesicle recruitment rates from reserve pools can be accelerated by neuromodulators modulators (serotonin) and that can affect the degree and time course of synaptic depression is similar to what has been proposed recently for temperature and phosphorylation effects at some synapses. In particular this issue has received recent and strong support from two papers looking at CNS and NMJ synapses [26,27].

There are several potential postsynaptic factors known in other models, such as receptor desensitization, receptor internalization, or spread of local mediators to neighboring synapses, which could account for synaptic depression. However, not all of these possibilities appear to be credible explanations to account for the results obtained in this study. The decay time of receptor desensitization is in the order of $5 \mathrm{msec}$ for glutamate receptors at the crayfish NMJ [28]. Stimulation at $5 \mathrm{~Hz}$ (i.e., $200 \mathrm{msec}$ delay) should be a sufficient time to remove any desensitization that occurs following each evoked stimulus. In addition, it should not be overlooked that facilitation occurs when the stimulation is increased from $0.5 \mathrm{~Hz}$ to $5 \mathrm{~Hz}$ and that it takes $\sim 20-40$ minutes of continuous $5 \mathrm{~Hz}$ stimulation to induce depression. Once the depression does start it proceeds at a rapid rate. Collectively, these results argue against the development of glutamate receptor desensitization as a mechanism of this homosynaptic depression. There remains the possibility that these qusiqualate-type receptors could behave in a similar manner as recently shown for AMPA receptors where rapid lateral migration in the postsynaptic membrane accounts for prevention of long term desensitization with mild use, but with high use could explain in part the longer time to recovery from depression [29]. This could explain a selective ef- fect of the phasic NMJ next to the tonic NMJ in the presented crayfish model. The local influence of synapses does not appear to occur in this system during synaptic depression because the synapses of the phasic and tonic neurons are in close association at many points along their innervation of a single muscle fiber and yet the tonic NMJ stays active after the phasic NMJs are depressed. Because no alteration in the amplitude of the EPSPs or in the short-term facilitation of the tonic NMJ was observed after depression of the phasic NMJs, this is evidence against potential diffusible factors from the phasic terminal. This result also argues against a retrograde signal from the muscle to the nerve terminal, unless a selective mechanism occurs to target only the phasic terminals in the midst of the tonic terminals.

It has been shown that the probability of release is greater at phasic NMJs than at tonic NMJs in this preparation [30] and this difference in sensitivity most likely accounts for the higher mean quantal content at phasic NMJs [30,22]. Also, the amount of the calcium binding protein, frequenin, is higher in phasic terminals than in tonic nerve terminals [31]. These results indicate that the amounts of available calcium and frequenin are quite different between the tonic and phasic terminals. In addition, ultrastructural analysis of phasic and tonic nerve terminals reveals that phasic nerve terminals contain a small number of single unbranched mitochondria, whereas a tonic terminal contains large numbers of multibranched mitochondria [21]. With such morphological and anatomical differences, one would predict that there are differences in the amount of available ATP in each type of nerve terminal. Thus, a phasic terminal could be more prone to ATP depletion due to fewer mitochondria influencing synaptic processes (e.g., vesicle recruitment, recycling) than a tonic nerve terminal. This high output terminal may even have enhance $\mathrm{Ca}^{2+}$ induce depression of the PMCA as compared to tonic terminals [32] or directly on the voltage gated $\mathrm{Ca}^{2+}$ channels [33].

Another possibility is that autoinhibition of the presynaptic terminal could account for some of the synaptic depression. The current working models for autoinhibition of glutamatergic synapses propose that kainate autoreceptors are involved. Recent studies in the hippocampus show that presynaptic kainate-sensitive receptors decrease the influx of calcium thereby reducing evoked transmission [34], and that this type of autoinhibition also involves the adenyl cyclase/protein kinase A pathway [35]. More recently, it was demonstrated that kainate receptors are located pre- and postsynaptically in the vertebrate CNS. Given that there are five different subunits of kainate receptors, a wide variety of responses to autoinhibition might be possible [36]. In fact, kainate-induced depression of cortical neurons is now assumed to occur due to the release of calcium from internal stores to produce long-term depression [36]. Such a possibility could occur at the crayfish NMJ. However, the working model of calcium dynamics in this preparation is that an increase in calcium release from internal stores results in enhanced transmission [37]. Recent evidence [38,39] suggests that presynaptic kainate receptors exist at the Drosophila NMJ, which is analogous to the crayfish NMJ in many ways. Conversely, presynaptic kainate receptors enhance the probability of vesicular fusion in Schaffer collateral synapses (mouse) [40]. In addition, an autoreceptor (via presynaptic mGluRs and calcium channels) has been confirmed to reduce 
the degree of short-term depression at large and small vertebrate synapses [41]. Similar situations may occur in the variation and density of presynaptic glutamatergic autoreceptor subtypes that control the net response to glutamate in crayfish motor nerve terminals. In fact, Schramm and Dudel [42] reported that activation of glutamatergic autoreceptors of the crayfish NMJ could result in either depression or excitation of transmission, and they proposed that the varying proportions of glutamatergic receptor subtypes could account for their observations.

It is established that vesicles can recycle empty at the Drosophila NMJ [43], so such a possibility might also occur at the crayfish NMJ. Even considering that Atwood et al., [44] saw a severe depletion of synaptic vesicles at crayfish NMJ after treatments that blocked ATP production and reduced presynaptic ATP levels, does not agree with ATP run down as the mechanism for depression since the action of 5HT relives depression. This was also shown in Logsdon et al., [7] in that glutamate depleted RRP vesicles were rapidly mixed with RP vesicles by 5-HT action.

ATP promotes NSF binding with $\alpha$-SNAP. ATP is required for undocking of vesicles via clathrin coated recycling [45]. A possible experiment to determine whether the mechanisms responsible for the rundown are related to the need for energy for vesicular docking and recycling (i.e., they are ATP-dependent processes) could be one in which the rapid release of caged ATP within depressed terminals is induced as was similarly approached with release of $\mathrm{Ca} 2+$ from caged compounds [46]. It would be interesting to examine, within this preparation, the possibility of the myosin light chain kinase induced inhibition of vesicular movement as shown in the Calyx of held synapse [47]. Comparative studies with NMJs in Drosophila that show marked synaptic depression might prove to be fruitful $[4,48]$.

In an earlier study of the crayfish opener muscle, Tabor and Cooper [49] demonstrated that approximately $80 \%$ of the enhancement of EPSPs could be blocked by 5-HT2 receptor antagonists in 5-HT activated NMJs. In addition, Dixon and Atwood [5] demonstrated that inhibiting inositol triphosphate blocked the rapid response of 5-HT in increasing the EPSP amplitude on the opener muscle of crayfish. These results raise questions on the types and densities of 5HT receptors present in phasic NMJs and whether they even possess the same pharmacological profiles as those of vertebrates. Past oversight in assuming cross-species similarities in pharmacology of serotonergic agents used in crustacean behavioral research has occurred [49,50]. The inconsistency in the results is probably due to the different intracellular cascades and receptor subtypes involved, as well as splicing differences for serotonin receptor subtypes $[50,51,52,53]$. To induce the formation of $\mathrm{IP}_{3}$ following stimulation of $5-\mathrm{HT}_{2}$ receptors it is possible that ATP is supplied by increasing the activity of phosphoinositide 3-kinase [54,55]. However, the fact that the depressed terminals were activated by 5-HT refutes the idea that a lack of ATP is responsible for the depression since enhanced vascular fusion takes place with 5HT exposure. If the enzymes associated with the vesicle's surface have the ability to generate ATP close to the vesicle, as shown for bovine neural tissue [56], then the proton ATP dependent pump may still function but a limiting factor would be to have enough glutamate present for the VGlut transporter to function with the sustained proton gradient. In addition, Lindgren and Smith [57] demonstrated that ATP production increased with electrical activity in crayfish motor neurons. At present, the mostly likely hypothesis to account for depression is that the rapidly recycling vesicles do not have time to repackage glutamate. The repackaging may even be slowed by some yet unknown inhibition of the glutamate transporter during high frequency stimulation. The effects of 5-HT do lend to the evidence for the 'pool' differentiation in recruitment. Despite that there are numerous possible mechanisms for depression (e.g. block of conduction in fine nerve branches, raised intracellular sodium if the sodium pump fails in a stressed terminal with low content of mitochondria, activation of inhibitory autoreceptors) these possibilities would not explain the rapid recovery induced by 5-HT in depressed terminals.

\section{ACKNOWLEDGEMENTS}

Appreciation is given to Hye Won Cooper for illustrative work. Funding was provided by a Howard Hughes Medical Institute undergraduate training fellowship (S.K.), a Ribble Fellowship, University of Kentucky, School of Biological Sciences (S.K., AFMJ) and two NSF grants, IBN-9808631 \& ILI DUE-9850907 (R.L.C.). Credit also is due to an undergraduate student, Dexter Reener, who did a one month internship in the laboratory as well to two high school science teachers (Lexington, KY), Ms. Heidi Anderson and Ms. Berry Hart. The high school teachers were supported by NSF-RET for a research internship. They helped with extensor preparations and vesicle analysis from TEM micrographs. The results of this study have previously been presented in abstract form [58,59].

\section{REFERENCES}

[1] Linden DJ, Conner JA. Long-term synaptic depression. Ann Rev Neurosci 1995; 18: 319-57.

[2] Atwood HL. Organization and synaptic physiology of crustacean neuromuscular systems. Prog Neurobiol 1973; 7: 291-391.

[3] Cooper RL, Stewart BA, Wojtowicz JM, Wang S, Atwood HL. Quantal measurement and analysis methods compared for crayfish and Drosophila neuromuscular junctions and rat hippocampus. J Neurosci Meth 1995; 61: 67-78.

[4] Atwood HL, Cooper RL. Synaptic diversity and differentiation: Crustacean neuromuscular junctions. Invert Neurosci 1996; 1: 291-307.

[5] Dixon D, Atwood HL. Conjoint action of phosphatidylinositol and adenylate cyclase systems in serotonin-induced facilitation at the crayfish neuromuscular junction. J Neurophysiol 1989; 62: 1251-9.

[6] Cooper RL, Dönmezer A, Shearer J. Intrinsic differences in sensitivity to 5-HT between high- and low-output terminals innervating the same target. Neurosci Res 2003; 45: 163-72.

[7] Logsdon S, Johnstone AFM, Viele K, Cooper RL. Regulation of synaptic vesicles pools within motor nerve terminals during short-term facilitation and neuromodulation. J Applied Physiol 2006; 100: 662-71.

[8] Wang C, Zucker RS. Regulation of synaptic vesicle recycling by calcium and serotonin. Neuron 1998; 21: 155-67.

[9] Dudel J. Facilitatory Effects of 5-Hydroxy-Tryptamine on the Crayfish Neuromuscular Junction. Naunyn Schmiedebergs Arch Exp Pathol Pharmakol 1965; 249: 515-28.

[10] Bradacs H, Cooper RL, Msghina M, Atwood HL. Differential physiology and morphology of phasic and tonic motor axons in a crayfish limb extensor muscle. J Exp Biol 1997; 200: 677-91.

[11] Atwood H L, Nguyen PV. Neural adaptation in crayfish. Am Zool 1995; 35: 28-36.

[12] Tse FW, Marin L, Jahromi SS, Atwood HL. Variation in terminal morphology and presynaptic inhibition at crustacean neuromuscular junctions. J Comp Neurol 1991; 304: 135-46.

[13] Cooper RL, Marin L, Atwood HL. Synaptic differentiation of a single motor neuron: conjoint definition of transmitter release, presynaptic calcium signals, and ultrastructure. J Neurosci 1995; 15: 4209-22. 
[14] Cooper RL, Winslow J, Govind CK, Atwood HL. Synaptic structural complexity as a factor enhancing probability of calcium-mediated transmitter release. J Neurophysiol 1996; 75: 2451-66.

[15] Atwood HL, Cooper RL. Assessing ultrastructure of crustacean and insect neuromuscular junctions. J Neurosci Meth 1996; 69: 51-8.

[16] Cooper RL, Harrington C, Marin L, Atwood HL. Quantal release at visualized terminals of crayfish motor axon: Intraterminal and regional differences. J Comp Neurol 1996; 375: 583-600.

[17] He P, Southard RC, Chen D, Whiteheart SW, Cooper RL. Role of alpha-SNAP in promoting efficient neurotransmission at the crayfish neuromuscular junction. J Neurophysiol 1999; 82: 3406-16.

[18] Sparks GM, Brailoiu E, Brailoiu GC, Dun NJ, Tabor J, Cooper RL. Effects of $\mathrm{m}-\mathrm{CPP}$ in altering neuronal function: blocking depolarization in invertebrate motor sensory neurons but exciting rat dorsal horn neurons. Brain Res 2003; 969: 14-26.

[19] Strawn JR, Neckameyer WS, Cooper RL. The effects of 5-HT on sensory neurons, CNS command, and neuromuscular junctions of the crayfish abdominal superficial flexor. Comp Biochem Physiol B 2000; 127: 533-50.

[20] Cooper RL, Hampson D, Atwood HL. Synaptotagmin-like expression in the motor nerve terminals of crayfish. Brain Res 1995; 703: 214-6.

[21] King MJ, Atwood HL, Govind CK. Structural features of crayfish phasic and tonic neuromuscular terminals. J Comp Neurol 1996; 372: 618-26.

[22] Msghina M, Millar AG, Charlton MP, Govind CK, Atwood HL. Calcium entry related to active zones and differences in transmitter release at phasic and tonic synapses. J Neurosci 1999;19: 8419-34.

[23] Rizzoli SO, Betz WJ. The structural organization of the readily releasable pool of synaptic vesicles. Science 2004; 303: 2037-39.

[24] Zampighi GA, Fain N, Zampighi LM, Cantele F, Lanzavecchia S, Wright, EM. Conical Electron Tomography of a Chemical Synapse: Polyhedral Cages Dock Vesicles to the Active Zone. J Neurosci 2008; 28(16): 4151-60.

[25] Lisman JE, Raghavachari S, Tsien RW. The sequence of events that underlie quantal transmission at central glutamatergic synapses. Nat Rev Neurosci 2007; 8(8):597- 609.

[26] Gaffield MA, Betz WJ. Synaptic vesicle mobility in mouse motor nerve terminals with and without synapsin. J Neurosci 2007; 27(50): 13691700 .

[27] Kushmerick C, Rendon R, von Gersdorf H. Physiological temperatures reduce the rate of vesicle pool depletion and short-term depression via an acceleration of vesicle recruitment. J. Neurosci 2006; 26(5):13661377.

[28] Dudel J, Franke C, Hatt H. Rapid activation and desensitization of transmitter-liganded receptor channels by pulses of agonists. Ion Channels 1992; 3: 207-60.

[29] Heine M, Groc L, Frischknecht R, Béïque JC, Lounis B, Rumbaugh G, Huganir RL, Cognet L, Choquet D. Surface mobility of postsynaptic AMPARs tunes synaptic transmission. Science 2008; 320(5873): 201-5.

[30] Msghina M, Govind CK, Atwood HL. Synaptic structure and transmitter release in crustacean phasic and tonic motor neurons. J Neurosci 1998; 18: 1374-82.

[31] Jeromin A, Shayan AJ, Msghina M, Roder J, Atwood HL. Crustacean frequenins: Molecular cloning and differential localization at neuromuscular junctions. J Neurobiol 1999; 41: 165-75.

[32] Scheuss V, Yasuda R, Sobczyk A, Svoboda K. Nonlinear [Ca2+] signaling in dendrites and spines caused by activity-dependent depression of Ca2+ extrusion. J Neurosci 2006; 26 (31): 8183-94.

[33] Mochida S, Few AP, Scheuer T, Catterall WA. Regulation of presynaptic $\mathrm{Ca}(\mathrm{V}) 2.1$ channels by $\mathrm{Ca} 2+$ sensor proteins mediates short-term synaptic plasticity. Neuron 2008; 57(2): 210-6.

[34] Kamiya H, Ozawa S. Kainate receptor-mediated presynaptic inhibition at the mouse hippocampal mossy fibre synapse. J Physiol 2003; 523: 653-65.

[35] Negrete-Diaz, JV, Sihra TS, Delgado-Garcia JM, Rodrquez-Moreno, A. Kainate receptor-mediated inhibition of glutamate release involves pro- tein kinase A in the mouse hippocampus. J Neurophysiol 2006; 96 : 1829-37.

[36] Park Y, Isaac JJ, Cho K. Long-term depression of kainate receptormediated synaptic transmission. Neuron 2006; 49: 95-106.

[37] Dropic AJ, Brailoiu E, Cooper RL. Presynaptic mechanism of action induced by 5 -HT in nerve terminals: Possible involvement of ryanodine and $\mathrm{IP}_{3}$ sensitive $\mathrm{Ca}^{2+}$ stores. Comp Biochem Phys A 2005; 142: 35561 .

[38] Bhatt D, Cooper RL. The pharmacological and physiological profile of glutamate receptors at the Drosophila larval neuromuscular junction. Phys Entomol 2005; 3: 305-10.

[39] Bhatt D, Bhatt D, Cooper RL. Characterization of glutamate receptors at the Drosophila neuromuscular junction. Society for Neuroscience annual meeting, Atlanta, GA 2006

[40] Sun HY, Dobrunz LE. Presynaptic kainate receptor activation is a novel mechanism for target cell-specific short-term facilitation at Schaffer collateral synapses. J Neurosci 2006; 26: 10796-807.

[41] von Gersdorff H, Schneggenburger R, Weis S, Neher E. Presynaptic depression at a calyx synapse: the small contribution of metabotropic glutamate receptors. J Neurosci 1997; 17(21): 8137-46.

[42] Schramm M, Dudel J. Metabotropic glutamate autoreceptors on nerve terminals of crayfish muscle depress or facilitate release. Neurosci Lett 1997; 234: 31-4.

[43] Kidokoro Y, Kuromi H, Delgado R, Maureira C, Oliva C, Labarca P. Synaptic vesicle pools and plasticity of synaptic transmission at the Drosophila synapse. Brain Res Rev 2004; 47: 18-32.

[44] Atwood HL, Lang F, Morin WA. Synaptic vesicles: selective depletion in crayfish excitatory and inhibitory axons. Science 1972; 176(41): 1353-5.

[45] Rothman JE. Intracellular membrane fusion. Adv Sec Mess Phosphoprotein Res 1994; 29: 81-96.

[46] Tsien RY, Zucker RS. Control of cytoplasmic calcium with photolabile tetracarboxylate 2-nitrobenzhydrol chelators. Biophys J 1986; 50: 84353.

[47] Srinivasan G, Kim JH, von Gersdorff H. The pool of fast releasing vesicles is augmented by Myosin light chain kinase inhibition at the calyx of held synapse. J Neurophysiol 2008; 99(4): 1810-24.

[48] Guo HF, Zhong Y. Requirement of Akt to mediate long-term synaptic depression in Drosophila. J Neurosci 2006; 26(15): 4004-14.

[49] Tabor JN, Cooper RL. Physiologically identified 5-HT2-like receptors at the crayfish neuromuscular junction. Brain Res 2002; 932: 91-8.

[50] Tierney AJ, Mangiamele LA. Effects of serotonin analogs on posture and agonistic behavior in crayfish. J Comp Physiol A 2001; 187: 75767.

[51] Southard RC, Haggard J, Crider ME, Whiteheart SW, Cooper RL. Influence of serotonin on the kinetics of vesicular release. Brain Res 2000; 871: 16-28.

[52] Kishore S, Stamm S. The snoRNA HBII-52 regulates alternative splicing of the serotonin receptor 2C. Science 2006; 311: 230-2.

[53] Krobert KA, Levy FO. The human 5-HT7 serotonin receptor splice variants: constitutive activity and inverse agonist effects. Br J Pharmacol 2005; 135: 1563-71.

[54] Carpenter CL, Duckworth BC, Auger KR, Cohen B, Schaffhausen BS, Cantley LC. Purification and characterization of phosphoinositide 3kinase from rat liver. J Biol Chem 1990; 255: 19704-11.

[55] Irvine RF. Inositol lipids in cell signaling. Curr Opin Cell Bio 1992; 4 212-9.

[56] Ikemoto A, Bole DG, Ueda, T. Glycolysis and glutamate accumulation into synaptic vesicles. J Biol Chem 2003; 278(8): 5929-40.

[57] Lindgren CA, Smith DO. Increased presynaptic ATP levels coupled to synaptic Activity at the crayfish neuromuscular junction. J Neurosci 1986; 6(9): 2644-52.

[58] Johnstone AFM, Kellie S, Reneer DV, Viele K, Cooper RL. Presynaptic depression in phasic motor nerve terminals and influence of 5-HT on docked vesicles. Soc Neurosci Abst 2005.

[59] Kellie SP, Cooper RL. Mechanisms of synaptic depression in high output phasic motor neurons. Amer Zool 1999; 39: 247A. 